\title{
Submitted: 18.11 .2020 Ultrasonography-guided sialolithotomy and stricture Accepted: dilations of the major salivary glands: a preliminary study
} 12.01.2021

Published: 16.08.2021

Keywords

endoscopic, assisted surgery, sialolith, submandibular gland, ultrasonography

\author{
Kaan Orhan ${ }^{1,2}$, Poyzan Bozkurt' ${ }^{3}$ Zeynep Serap Berktaş ${ }^{1}$, \\ Mehmet Hakan Kurt ${ }^{1}$ \\ ${ }^{1}$ Department of Dentomaxillofacial Radiology, Ankara University Faculty of Dentistry, Turkey \\ ${ }^{2}$ Medical Design Application and Research Center (MEDITAM), Ankara University, Turkey \\ ${ }^{3}$ Department of Oral and Maxillofacial Surgery, Ankara University Faculty of Dentistry, Turkey \\ Correspondence: Dr. Mehmet Hakan Kurt,e-mail:mhakankurt@yahoo.com
}

DOI: $10.15557 / \mathrm{JoU} .2021 .0038$

\begin{abstract}
Aim of the study: The present preliminary study aims to evaluate the possible positive outcomes of ultrasonography-guided sialolithotomies and duct stricture dilations utilizing stone retrieval baskets and guide wires. Case description: A total of 6 cases in an ongoing study (4 cases of sialolithiasis and 2 cases of duct strictures with intraluminal adhesion) were analyzed. All sialoliths were $<5 \mathrm{~mm}$ in diameter. Stone removals and duct dilations were performed under ultrasonography guidance with two different types of linear probes. Edema measurements were carried out, and the area of edema was evaluated via the echogenicity changes. Patient satisfaction was also evaluated by the patients themselves using a Visual Analog Scale questionnaire on postoperative day 0 , and on days 1, 2, and 3. Conclusions: There were no postoperative complications, and mouth openings returned to normal at 7-day follow-up. The pain scores decreased after 6 hours, and pain subsided completely after 12 hours in all the patients. Edema also resolved gradually after the operation. The patient satisfaction levels were high.
\end{abstract}

\section{Introduction}

Sialolithiasis (salivary calculi or salivary stones) is a condition in which a calcified mass forms within a salivary gland, usually in the submandibular gland duct (Wharton's duct). Predisposition to the condition is linked to anatomical and physiological factors. Sialolithiasis is one of the most common reasons for head and neck swelling, and one of the major diseases involving the salivary glands. Patients usually present with oral discomfort during eating, episodic swelling and fullness peri- and postprandially during times of salivary gland stimulation ${ }^{(1)}$.

Surgical removal of the sialolithiasis is often necessary due to recurrent symptoms. In recent years, though, the surgical treatment of submandibular gland sialolithiasis has shifted away from traditional surgery (sialoadenectomy) toward gland-preserving, minimally invasive approaches including lithotripsy, endoscopic removal using graspers, and retrieval with a basket under fluoroscopic control(2).

Stone retrieval with baskets is one of the minimally invasive methods which are easy to access and cost efficient. Although the retrieval of stones with baskets is reported to

Tab. 1. Patient demographics and sialolith data

\begin{tabular}{|c|c|c|c|c|}
\hline & Gender & Age & Location & Diameter \\
\hline Case 1 & Male & 47 & SGC, horizontal portion & $4.6 \mathrm{~mm}$ \\
\hline Case 2 & Male & 47 & SGC, horizontal portion & $3.46 \mathrm{~mm}$ \\
\hline Case 3 & Male & 58 & SGC & $3.36 \mathrm{~mm}$ \\
\hline Case 4 & Female & 39 & SGC & $4.18 \mathrm{~mm}$ \\
\hline Case 5 & Male & 48 & SGC & Duct strictures \\
\hline Case 6 & Female & 42 & SGC & Duct strictures \\
\hline
\end{tabular}



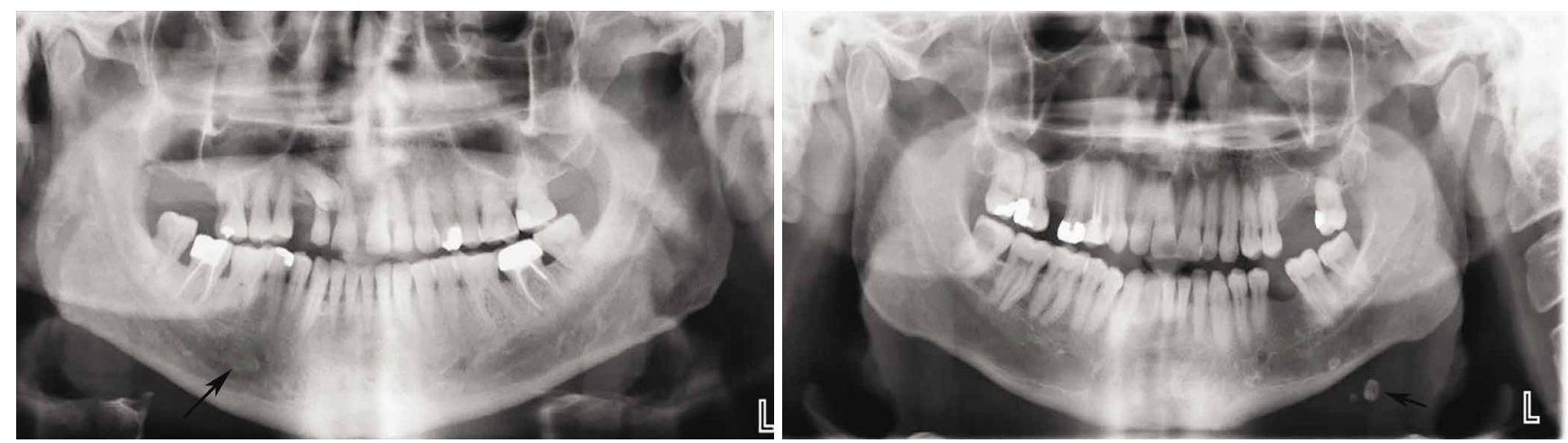

Fig. 1. Panoramic radiographs of two cases (sialoliths indicated with black arrows)


Fig. 2. Application of Introducer kit $4 F$ and Triangular Nitinol stone retrieval device

require fluoroscopic guidance, successful basket retrieval under ultrasound guidance has also been reported ${ }^{(3)}$.

To the best of the authors' knowledge, there are no studies or case series presenting postoperative complications or satisfaction analyses after sialolith removal performed with this method. The presented case series reports the application of both extraoral and intraoral ultrasound probes to guide the removal of a submandibular duct calculus with a stone retrieval basket. The cases were analyzed to determine complications including infection, bleeding, edema, and maximum mouth opening, as well as patient satisfaction rates.

\section{Material and methods}

The study was approved by the Ankara University Faculty of Dentistry Ethics Committee. Written consent has been obtained from all the patients.

In total, there were 6 cases in an ongoing study (Tab. 1), with 4 diagnosed with sialolithiasis based on panoramic/ occlusal radiographs, and 2 with a further diagnosis of salivary duct strictures with intraluminal adhesion based on USG findings (Fig. 1 and Fig. 2). All the conditions involved the submandibular gland, and all the patients were symptomatic, presenting with edema and xerostomia.

A senior dentomaxillofacial radiologist with 15 years' experience in ultrasonography was responsible for the ultrasound diagnosis in all the cases. Stone removals with baskets were planned under ultrasonography guidance using ACUSON S 2000 (Siemens, Germany) unit with two different types of linear probes. The linear probe that was used intraorally (IOP) was a 14L5 SP Transducer probe, while a 9L4 Transducer probe (Siemens, Germany) was used extraorally (EOP). The IOPs were covered with sterile surgical hand gloves for intraoral applications, and the gel was placed inside the surgical hand gloves. Stricture openings and duct dilations were made via an intraductal approach from natural orifices with Introducer kits 4F and 5F (Shanghai Kindly Enterprise, Shanghai, China). After dilations were achieved, 1.9F Dakota Triangular Nitinol stone retrieval devices (Boston Scientific, Indiana, USA) were introduced into the canals to retrieve the sialoliths.

While the baskets were in a closed status, the sialoliths were bypassed (Fig. 3), and the baskets were then opened via the handle, and slowly retracted to entrap the sialoliths in the basket device. Several attempts were needed 


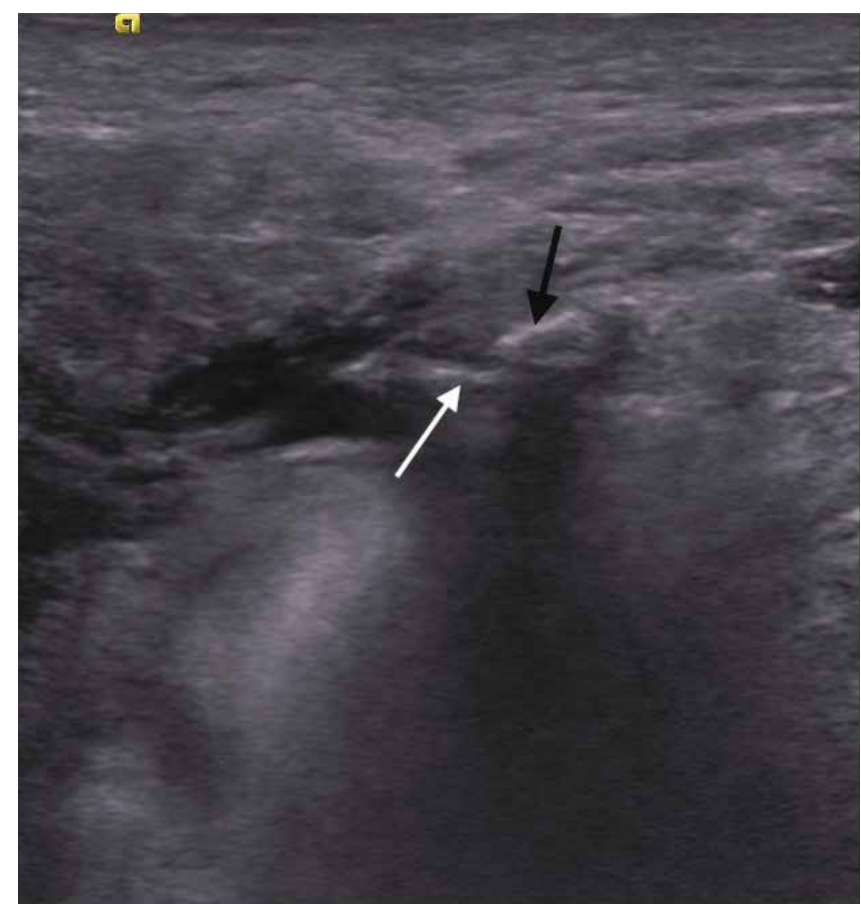

Fig. 3. USG image of retrieval basket bypassing the sialolith (black arrow indicates the sialolith, white arrow indicates the retrieval basket) before the procedure was successful. After the entrapment of sialoliths, the baskets were closed via the handle, and the entrapped sialoliths were slowly removed from the canals. All steps of the dilation and stone retrieval procedures were guided by ultrasound, and the stones and basket devices were clearly visualized during all the procedures (Fig. 4).

No local anesthesia was needed for the procedures. The patients were instructed to alert the operator if pain occurred, although none requested anesthesia.

Edema measurements were done extra- and intraorally on days 1 and 2 by the same senior dentomaxillofacial radiologist. The patients' heads were kept in a neutral, fixed position in a custom-made head stabilizer. For extraoral measurements, the EOP was placed submental on the midsagittal plane, centered between the anterior border of the hyoid bone and the posterior border of the mandibular symphysis, with the central sound wave running perpendicular to the Frankfort horizontal plane. For intraoral measurements, the patients' heads were also kept in a neutral, fixed position in a custom-made head stabilizer. The IOP was placed intraorally on the mouth floor in maximum mouth opening.

B-mode scanning was used to identify edema and thickening of the subcutaneous (extraoral measurement) and submucosal layers (intraoral measurement). The area of edema was

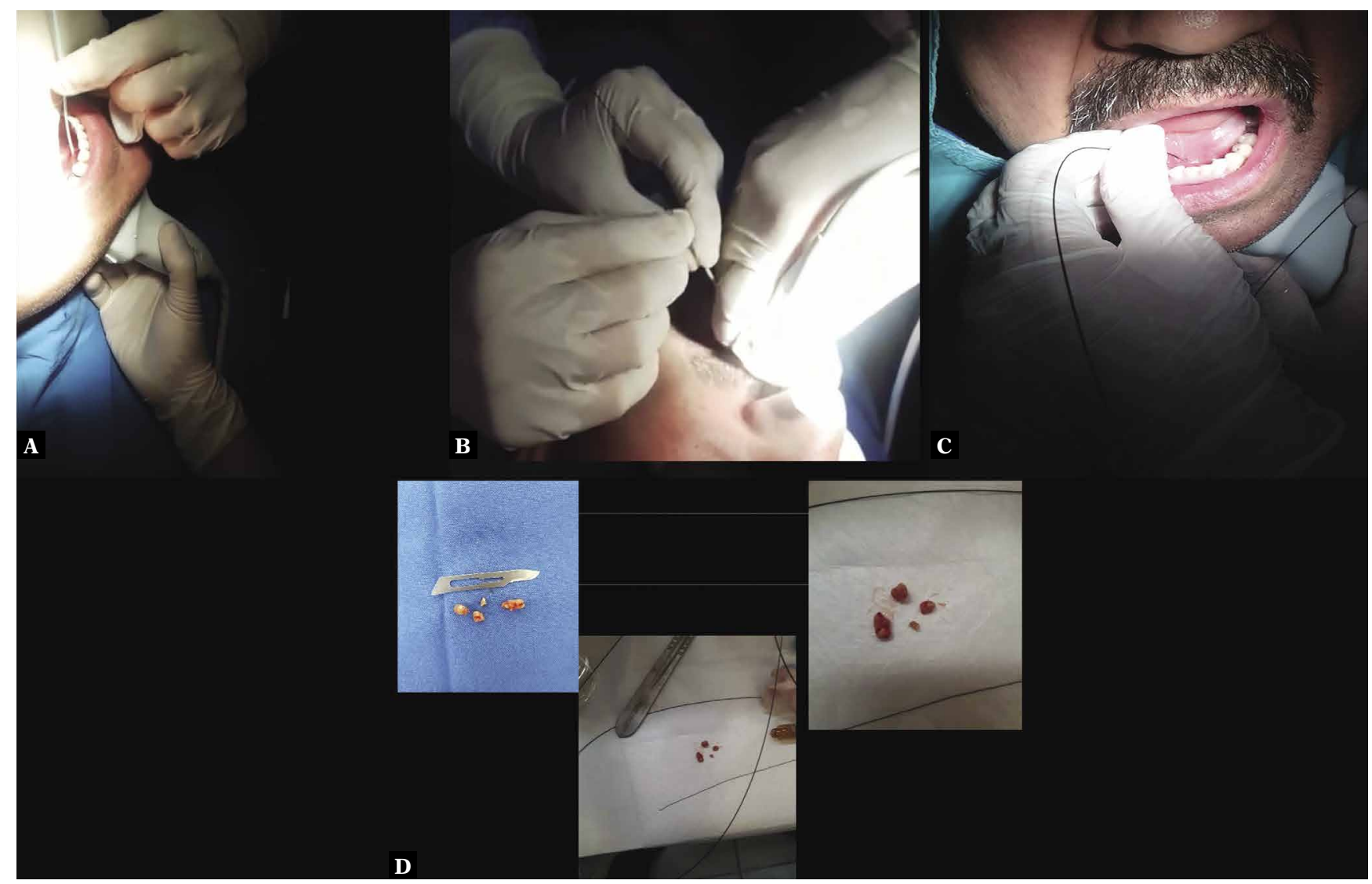

Fig. 4. Insertion of the guide wire under USG guidance with extraoral transducer (A), Insertion of the guide wire under USG guidance with intraoral transducer (B), advancing the retrieval basket through the submandibular duct (C), clinical appearance of retrieved sialoliths (D) 



Fig. 5. USG images showing subcutaneous edema measurements

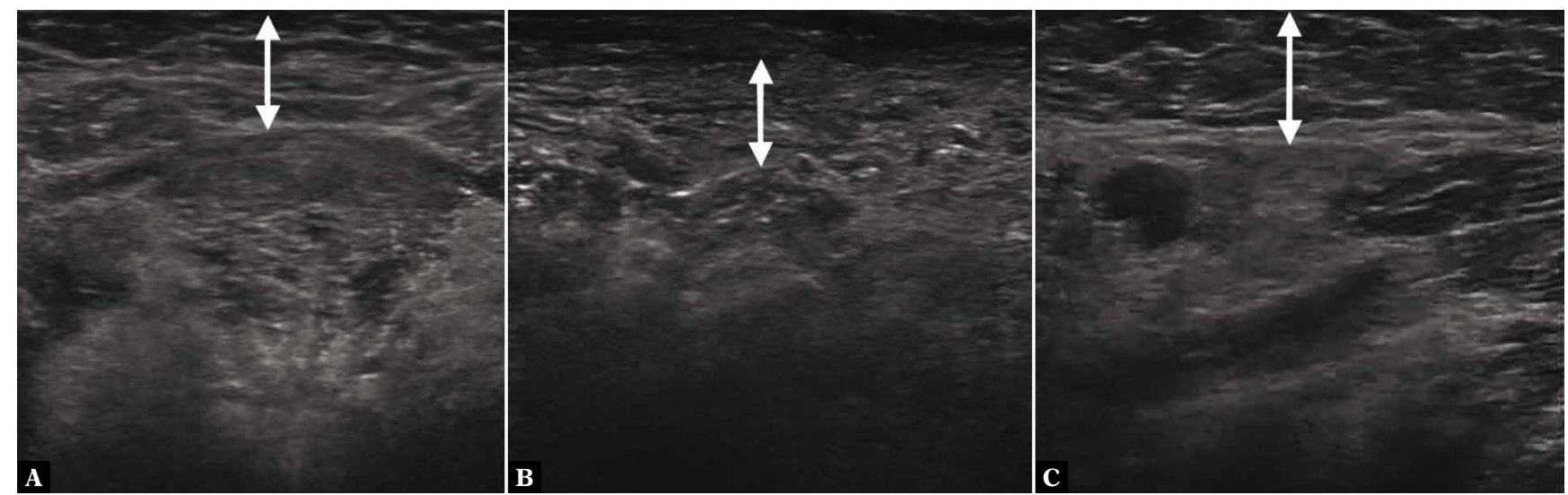

Fig. 6. Definition of subcutaneous echogenicity A. Grade 0, B. Grade 1, and C. Grade 2

calculated via the echogenicity changes (Fig. 5). Edema was defined as echogenicity of the isoechoic tissues similar to the unaffected site but with an increase in fluid content. The echogenicity was classified as Grade 0: no increased echogenicity, Grade 1: diffuse increases in echogenicity, but identifiable horizontal or obliquely oriented echogenic lines caused by connective tissue bundles, Grade 2: diffuse increases in echogenicity, echogenic lines are not identifiable (Fig. 6). Since echogenicity is a relative evaluation, adjustments using normal subcutaneous fat from another part of the body were made.

The patients themselves evaluated the severity of pain with a Visual Analog Scale (VAS) questionnaire, both preoperatively and postoperatively at 6 and 12 hours, and on days 1,2 , and 3 . Mouth opening was evaluated by measuring the distance between the upper and lower incisors on maximum mouth opening with a rigid-ruler, both preoperatively, and on postoperative days 1 and 2. Postoperative complications and other complaints were noted during follow-up visits. Patient satisfaction was evaluated by the patients themselves with a VAS questionnaire on postoperative day 0 , and on days 1,2 , and 3 .

\section{Results}

Patient demographics and sialolith data are presented in Tab. 1. The stone sizes varied from a minimum of $3.36 \mathrm{~mm}$ to $4.6 \mathrm{~mm}$.

Pain scores, mouth-opening, postoperative complications, and patient satisfaction data are presented in Tab. 2. All the results were considered satisfactory. The pain subsided completely after 12 hours in all the patients. There were no postoperative complications. The satisfaction scores among the patients were high (8-10) starting from immediate postoperative evaluations for USG-guided retrieval operation.

Postoperatively, only diffuse increases in subcutaneous echogenicity were observed during the postoperative days 1 and 2 in all the cases (Tab. 3). 
Tab. 2. Pain scores, mouth-opening measurements, postoperative complications and patient satisfaction levels

\begin{tabular}{|c|c|c|c|c|c|c|c|}
\hline & Time & Case 1 & Case 2 & Case 3 & Case 4 & Case 5 & Case 6 \\
\hline \multirow{6}{*}{ Pain (VAS) } & Preoperative & 5 & 6 & 4 & 4 & 3 & 2 \\
\hline & $6^{\text {th }}$ hour & 2 & 2 & 0 & 1 & 0 & 0 \\
\hline & $12^{\text {th }}$ hour & 0 & 1 & 0 & 0 & 0 & 0 \\
\hline & $1^{\text {st }}$ day & 0 & 0 & 0 & 0 & 0 & 0 \\
\hline & $2^{\text {nd }}$ day & 0 & 0 & 0 & 0 & 0 & 0 \\
\hline & $3^{\text {rd }}$ day & 0 & 0 & 0 & 0 & 0 & 0 \\
\hline \multirow{3}{*}{ Mouth opening $(\mathrm{cm})$} & 0 hour & 4.7 & 4.5 & 5.2 & 4.8 & 5.1 & 4.6 \\
\hline & $1^{\text {st }}$ day & 4.7 & 4.2 & 5.2 & 4.8 & 5.0 & 4.7 \\
\hline & $2^{\text {nd }}$ day & 4.7 & 4.5 & 5.2 & 4.8 & 5.2 & 4.7 \\
\hline \multirow{5}{*}{ Postoperative complication } & $6^{\text {th }}$ hour & - & - & - & - & - & - \\
\hline & $12^{\text {th }}$ hour & - & - & - & - & - & - \\
\hline & $1^{\text {st }}$ day & - & - & - & - & - & - \\
\hline & $2^{\text {nd }}$ day & - & - & - & - & - & - \\
\hline & $3^{\text {rd da }}$ day & - & - & - & - & - & - \\
\hline Other & & - & - & - & - & - & - \\
\hline \multirow{4}{*}{ Satisfaction (VAS) } & 0-hour (immediate postoperative) & 10 & 8 & 10 & 10 & 10 & 10 \\
\hline & $1^{\text {st }}$ day & 10 & 10 & 10 & 10 & 10 & 10 \\
\hline & $2^{\text {nd }}$ day & 10 & 10 & 10 & 10 & 10 & 10 \\
\hline & $3^{\text {rd }}$ day & 10 & 10 & 10 & 10 & 10 & 10 \\
\hline
\end{tabular}

Subcutaneous edema measurements are presented in Tab. 4. Although edema increased following the operations on day 1, after day 2 edema decreased gradually. The patients were recalled for their 7-day follow-up. No edema was detected during a clinical examination.

\section{Discussion}

Sialolithiasis (salivary calculi or salivary stone) refers to a calcified mass formed in the salivary gland. It is a rare condition, affecting 1 per 10 000-300 000 (3). Symptoms such as swelling and pain may occur. These symptoms increase especially when saliva flow is triggered ${ }^{(3)}$.

Ultrasound, routine x-rays, sialography, and computed tomography are used in the diagnosis of salivary gland stones. Ultrasound is a non-invasive, less costly, and easily accessible method, and it is used not only to diagnose the stones and determine their location, but also as an aid in accessing them ${ }^{(3)}$.

The stones can be removed spontaneously with the flow of saliva, but if they are not, they may need to be surgically removed. Since total removal of the submandibular gland may result in nerve damage (at the rate of $8 \%$ ), postoperative saliva flow and amount may cause problems, and structural changes may occur in the gingival epithelium in case of gland deficiency, surgical removal of the submandibular gland is only indicated in cases where the stone is located within the gland ${ }^{(3)}$.

Today, minimally invasive methods are typically preferred in cases that do not require the use of conventional surgical methods. Sialoendoscopy is the method used to access and remove these stones. This method is non-invasive, and provides direct access to the canal, making it possible to
Tab. 3. Subcutaneous echogenicity measurements

\begin{tabular}{|c|c|}
\hline Subcutaneous echogenicity & Grade \\
\hline \multicolumn{2}{|c|}{ Patient 1 (Sialolithotomy) } \\
\hline Preoperative & 0 \\
\hline Postoperative $1^{\text {st }}$ day & 1 \\
\hline Postoperative $2^{\text {nd }}$ day & 1 \\
\hline \multicolumn{2}{|c|}{ Patient 2 (Sialolithotomy) } \\
\hline Preoperative & 0 \\
\hline Postoperative $1^{\text {st }}$ day & 1 \\
\hline Postoperative $2^{\text {nd }}$ day & 1 \\
\hline \multicolumn{2}{|c|}{ Patient 3 (Sialolithotomy) } \\
\hline Preoperative & 0 \\
\hline Postoperative $1^{\text {st }}$ day & 0 \\
\hline Postoperative $2^{\text {nd }}$ day & 1 \\
\hline \multicolumn{2}{|c|}{ Patient 4 (Sialolithotomy) } \\
\hline Preoperative & 1 \\
\hline Postoperative $1^{\text {st }}$ day & 1 \\
\hline Postoperative $2^{\text {nd }}$ day & 1 \\
\hline \multicolumn{2}{|c|}{ Patient 5 (Duct stricture dilation) } \\
\hline Preoperative & 0 \\
\hline Postoperative $1^{\text {st }}$ day & 1 \\
\hline Postoperative $2^{\text {nd }}$ day & 1 \\
\hline \multicolumn{2}{|c|}{ Patient 6 (Duct stricture dilation) } \\
\hline Preoperative & 0 \\
\hline Postoperative $1^{\text {st }}$ day & 0 \\
\hline Postoperative $2^{\text {nd }}$ day & 0 \\
\hline
\end{tabular}

dilate and irrigate the ductal system. However, there are also limiting factors such as stone size, shape, orientation, and location within the salivary gland canal in surgeries performed using only the endoscope ${ }^{(4)}$. These challenging situations may arise from the thickness of the endoscope itself, and this factor can also reduce the level of patient satisfaction by increasing postoperative complaints such 
as pain and edema. In addition to these factors, there are other disadvantages including high cost of equipment and difficult access. Based on these considerations, the current study investigates stone retrieval under ultrasound guidance only.

In the literature, endoscopic interventions to the salivary gland are indicated as a new trend, and success rates as high as $82 \%$ have been reported. However, it is stated that this success rate can decrease to as low as $35 \%$ for stones larger than $3 \mathrm{~mm}^{(4)}$. Salivary stones should be managed according to the size, location, shape, number, impaction, and mobility of sialoliths ${ }^{(2)}$. The salivary stone sizes in the present study varied from $3.36 \mathrm{~mm}$ to $4.6 \mathrm{~mm}$. The results of the present study showed no unsuccessful interventions and high patient satisfaction rates. These different results in success can be attributed to the difficulty in bypassing the stones through the endoscope and manipulation during the removal of the stones due to the thickness of the endoscope and its rigid structure.

Another guiding method used in salivary gland stone retrieval is fluoroscopy. However, high levels of radiation are received by the patient due to simultaneous x-ray use, and there is a risk of allergic reactions caused by iodine used in contrast materials. For these reasons, it may be considered inferior to other methods ${ }^{(2)}$.

Drage and McAuliffe described the ultrasound-guided basket retrieval method ${ }^{(2)}$. They reported the frequency of the probe as one of the factors affecting the success of the procedure and they used a linear 7.0-10 MHz probe in their study. They reported these data as "ideal", as the method achieved a sufficient resolution and penetration depth. However, they added that higher frequencies will increase the resolution while affecting the penetration depth. In the present study, two different USG units with IOP (4-13 MHz, 14L5 SP Transducer) and EOP (5-13.3 MHz, 9L4 Transducer) probes were used. Although the present study did not investigate the efficiency of the probes used, ease of use was reported both by the dentomaxillofacial radiologist and the surgeon. Rogers and Drage reported the retrieval of a submandibular duct stone in a 13-year-old boy, and highlighted that the technique may have an advantage over the conventional surgical techniques in pediatric patients ${ }^{(5)}$. The two case reports using the same ultrasoundguided basket retrieval method as in the present study did not report postoperative complications nor patient satisfaction, thus no comparison could be made without relevant data $(2,5)$.

Reported postoperative complications after sialoadenectomy include permanent nerve damage (1.4-3.3\%), hemorrhage $(0-14 \%)$, fistulae formation $(0-4 \%)$, infection $(0-14 \%)$, cyst $(0-3 \%)$, and scar formation $(0-16 \%)^{(6)}$. Makdissi et al. evaluated the intraoral surgical removal of sialoliths from the hilum of the submandibular gland. The glands of four patients developed repeated infections during the follow-up and had to be removed surgically ${ }^{(7)}$. Nevertheless, in the study by Juul et al. the success rate as high as $93 \%$ and a high patient satisfaction level (94\%) have
Tab. 4. Subcutaneous edema measurements

\begin{tabular}{|c|c|c|}
\hline Edema measurement & Intraoral $\left(\mathbf{m m}^{2}\right)$ & Extraoral $\left(\mathrm{mm}^{2}\right)$ \\
\hline \multicolumn{3}{|c|}{ Case 1 (Sialolithotomy) } \\
\hline Preoperative (I) & 0.34 & 0.68 \\
\hline Postoperative $1^{\text {st }}$ day (II) & 0.48 & 1.09 \\
\hline Postoperative $2^{\text {nd }}$ day (III) & 0.28 & 0.81 \\
\hline \multicolumn{3}{|c|}{ Case 2 (Sialolithotomy) } \\
\hline Preoperative (I) & 0.68 & 1.03 \\
\hline Postoperative $1^{\text {st }}$ day (II) & 0.78 & 1.2 \\
\hline Postoperative $2^{\text {nd }}$ day (III) & 0.69 & 1.07 \\
\hline \multicolumn{3}{|c|}{ Case 3 (Sialolithotomy) } \\
\hline Preoperative (I) & 0.35 & 0.84 \\
\hline Postoperative $1^{\text {st }}$ day (II) & 0.45 & 1.28 \\
\hline Postoperative $2^{\text {nd }}$ day (III) & 0.36 & 0.98 \\
\hline \multicolumn{3}{|c|}{ Case 4 (Sialolithotomy) } \\
\hline Preoperative (I) & 0.84 & 0.92 \\
\hline Postoperative $1^{\text {st }}$ day (II) & 1.18 & 1.28 \\
\hline Postoperative $2^{\text {nd }}$ day (III) & 1.05 & 1.05 \\
\hline \multicolumn{3}{|c|}{ Case 5 (Duct stricture dilation) } \\
\hline Preoperative (I) & 0.58 & 0.90 \\
\hline Postoperative $1^{\text {st }}$ day (II) & 0.78 & 1.10 \\
\hline Postoperative $2^{\text {nd }}$ day (III) & 0.62 & 0.99 \\
\hline \multicolumn{3}{|c|}{ Case 6 (Duct stricture dilation) } \\
\hline Preoperative (I) & 0.18 & 0.40 \\
\hline Postoperative $1^{\text {st }}$ day (II) & 0.38 & 0.60 \\
\hline Postoperative $2^{\text {nd }}$ day (III) & 0.20 & 0.51 \\
\hline
\end{tabular}

been reported after the treatment of sialolithiasis by transoral incision of Warthon's duct ${ }^{(8)}$. Their patients were assessed on a quality-of-life scale, and $71 \%$ of them experienced no post-surgical swelling of the gland, yet scar tissue, tingling, and decreased sensation of the tongue was also reported. In the present study, a similar result was obtained concerning swelling, yet the evaluation was performed by ultrasound, a comparably more objective method. The authors also reported that $92 \%$ of patients had no symptoms, and in $94 \%$ of the cases the glands were preserved, and there were no infections, although one patient $(3 \%)$ suffered from damage to the lingual nerve, and $6 \%$ claimed to suffer from continuous tingling of the tongue. Combes et al. investigated the intraoral surgical removal of proximal submandibular stones and reported similar results. All sialoliths except one were retrieved successfully; in addition, there were no peri- or early postoperative complications. Nevertheless, $6 \%$ had a persistent tingling sensation of the tongue. Patient satisfaction was high, estimated at $92 \%$.

In a study conducted via the lithotripsy/sialoendoscopy approach, no immediate postoperative complications were reported. Nevertheless, one patient underwent gland excision for retained stone and chronic sialadenitis, and postoperatively suffered a transient hypoglossal and lingual nerve weakness. Another patient underwent repeated sialolithotomy for retained sialolith and the last notable complication was salivary gland fistula ${ }^{(10)}$. Similarly, Lee et al., in their study using the sialoendoscopy method, also reported high patient satisfaction levels with no reported postoperative complications ${ }^{(3)}$. 


\section{Conclusion}

In the present study, the pain scores, mouth-opening, postoperative complications, and patient satisfaction results were found to be good, with no unsuccessful interventions. Based on the results, the USG-guided basket retrieval approach for sialolithotomies and duct stricture dilations involving the submandibular gland seems to be a satisfactory method, ensuring high levels of patient satisfaction. The approach may be suggested in the settings where equipment required for sialoendoscopy and lithotripsy is not available.

\section{References}

1. Delli K, Spijkervet FK, Vissink A: Salivary gland diseases: infections, sialolithiasis and mucoceles. Monogr Oral Sci 2014; 24: 135-148.

2. Drage NA, McAuliffe NJ.:Ultrasound-guided basket retrieval of salivary stones: a new technique. Brit J Oral Maxillofac Surg 2005; 43: 246-248.

3. Lee D-K, Kim E-H, Kim C-W, Kang M-H, Song I-S, Jun S-H: Sialolithotomy of the submandibular duct using sialendoscopy. Maxillofac Plast Reconstr Surg 2019; 41: 24.

4. Joshi AS, Sood AJ: Ultrasound-guided needle localization during open parotid sialolithotomy. Otolaryng Head Neck Surg 2014; 151: 59-64.

5. Rogers DJ, Drage NA: Ultrasound-guided basket retrieval of a submandibular duct stone in a child. J Pediatr Surg 2006; 41: 1780-1782.

6. McGurk M, Makdissi J, Brown JE: Intra-oral removal of stones from the hilum of the submandibular gland: report of technique and morbidity. Int J Oral Maxillofac Surg 2004; 33: 683-686.

\section{Acknowledgments}

The procurement of the Introducer kits and Triangular Nitinol stone retrieval devices used in the study was financed by Ankara University Scientific Research Projects.

\section{Conflict of interest}

Authors do not report any financial or personal connections with other persons or organizations which might negatively affect the contents of this publication and/or claim authorship rights to this publication..

7. Makdissi J, Ezcudier MP, Brown JE, Osailan S, Drage N, McGurk M: Glandular function after intraoral removal of salivary calculi from the hilum of the submandibular gland. Brit J Oral and Maxillofac Surg 2004; 42: 538-541.

8. Juul ML, Wagner N: Objective and subjective outcome in 42 patients after treatment of sialolithiasis by transoral incision of Warthon's duct: a retrospective middleterm followup study. Eur Arch Otorhinolaryngol 2014; 271: 3059-3066.

9. Combes J, Karavidas K, McGurk M: Intraoral removal of proximal submandibular stones - an alternative to sialadenectomy? Int J Oral Maxillofac Surg 2009; 38: 813-816.

10. Philips J, Withrow K: Outcomes of holmium laser-assisted lithotripsy with sialendoscopy in treatment of sialolithiasis. Otolaryngol Head Neck Surg 2014; 150: 962-967. 